\title{
Successful Treatment of Dermatophytomas in 19 Patients Using Efinaconazole $10 \%$ Solution
}

\author{
Casey Wang Wendy Cantrell Theresa Canavan Boni Elewski \\ University of Alabama at Birmingham, Department of Dermatology, Birmingham, AL, USA
}

\section{Keywords \\ Fungal infection · Nail disorder · Nail fungus . \\ Onychomycosis}

\begin{abstract}
Dermatophytomas represent a historically difficult-to-treat complication of onychomycosis and are characterized by adherent fungal masses encased in biofilm in the subungual space. In this study, we evaluated the efficacy of $10 \%$ efinaconazole solution in patients with onychomycosis complicated by dermatophytomas. Cure was achieved in $65 \%$ of target great toenails. All dermatophytomas resolved and did not recur during the study. The median time to dermatophytoma resolution was 16 weeks.
\end{abstract}

(C) 2019 S. Karger AG, Basel

\section{Introduction}

Onychomycosis is a fungal infection of the nail unit generally caused by dermatophyte fungi, which can result in discoloration, subungual debris, nail plate thickening, and onycholysis. Onychomycosis is associated with older age and comorbidities that impair immunity and circula- tion in the lower legs, such as diabetes mellitus, peripheral vascular disease, and solid organ or bone marrow transplants. The most common type of onychomycosis is distal, lateral subungual onychomycosis (DLSO). In younger individuals, DLSO may occur as a result of trauma, particularly if the patient is exposed to conditions which potentiate fungal growth, such as frequent use of athletic gear and public swimming pools [1]. In DLSO, the infection involves the subungual space and may extend from the hyponychium proximally until the nail matrix is involved in advanced cases.

Historically, onychomycosis therapy required prolonged courses of systemic antifungal therapy such as terbinafine or itraconazole [1]. Effective topical therapy for DLSO has been challenging due to the location of infection under and within the nail plate [2]. An effective therapeutic agent must be able to diffuse through the nail plate and remain at high enough antimicrobial concentrations in the subungual space. Recently, there have been new antifungal solutions for topical treatment designed to penetrate the nail plate and spread in the subungual space [3].

Despite recent advances in topical therapies for onychomycosis, little is known about their role in DLSO complicated by dermatophytomas. Dermatophytomas 
appear as yellow or white streaks or patches in the subungual space and are dense fungal masses encased in a layer of biofilm, and they are less responsive to systemic antifungal therapy [4]. Historically, patients with dermatophytomas have been excluded from most clinical trials of topical and oral drugs $[3,5]$. There has been one case report of dermatophytoma resolution with daily efinaconazole application in a patient who failed oral terbinafine and fluconazole [6].

In an open-label study, we treated 19 patients (20 target nails) with DLSO complicated by a dermatophytoma with daily application of efinaconazole $10 \%$. Diagnosis was made based on nail appearance plus mycology confirmation at the University of Texas Health Science Center lab.

\section{Methods}

Patients with onychomycosis were screened according to the following inclusion criteria: age $\geq 18$ years, provision of informed consent, presence of a dermatophytoma on one or both great toenails, target great toenail thickness $\leq 3.0 \mathrm{~mm}$, and having either positive potassium hydroxide $(\mathrm{KOH})$ showing septate hyphae on microscopy or being culture positive for a dermatophyte fungus. Subjects were excluded based on history of immunosuppression by disease or therapy, history of psoriasis or other nail disease, three or more dermatophytomas on the target nail, and unwillingness to avoid nail polish during the study and follow-up period. The central mycology lab used was the University of Texas Health Science Center in San Antonio, TX. One patient was enrolled on clinical grounds alone. Two patients enrolled had negative cultures at baseline but were culture positive for a dermatophyte in a previous study, on which they failed treatment.

Upon study qualification, patients were given efinaconazole $10 \%$ solution along with written instructions to apply daily on, under, and around all affected toenails. Patients were evaluated in clinic at weeks 0 (baseline), 4, 8, and every 8 weeks thereafter for 1 year. Onychomycosis severity scoring (OASIS) and photography of the target nail were done at each visit, and nail cultures and microscopy were done at baseline and repeated at specified intervals [7]. Subject compliance was assessed using take-home paper calendars on which subjects checked off daily applications.

The primary efficacy endpoints were the clinical resolution of dermatophytomas, as well as cure defined as completely normal nail plate or negative fungal culture and microscopy plus less than $10 \%$ residual onycholysis without evidence of distal subungual hyperkeratosis or nail plate thickening at end of study [8]. For subjects who withdrew early from the study or were lost to follow-up but still completed at least 48 weeks of treatment, the last fungal culture at week 32 was used for analysis, provided that less than $10 \%$ of the nail was affected by onycholysis and the target nail did not have other signs of onychomycosis at the time of culture.

Successful Treatment of Dermatophytomas with Efinaconazole 10\%

\section{Results}

Nineteen subjects and 20 great toenails were included in the data analysis. Subjects ranged in age from $37-76$ years. Seven females and 13 males were enrolled, and one female was not included in the final analysis due to early termination after 2 months of therapy. One of the subjects included had dermatophytomas on both great toenails. All included subjects were treated with efinaconazole $10 \%$ solution daily for at least 48 weeks. Early termination occurred in two subjects who met definition of cure by week 48 of treatment (by achieving negative culture and clear target nail [ $0 \%$ involvement]). One subject terminated early at week 48 after no significant improvement was seen beyond the resolution of the dermatophytoma. Another subject terminated electively at end of treatment due to desire to begin wearing nail polish. Three subjects were lost to follow-up after week 48 but are included in the analysis. Treatment-related adverse events included ingrown toenails, which occurred in previous studies as well. No severe adverse events occurred, and no adverse events necessitating discontinuation from therapy occurred. No significant problems with compliance were observed.

At baseline, 90\% (18/20) nails had positive microscopy $(\mathrm{KOH})$ results at the central lab, indicative of fungal infection. All patients presented with the clinical symptoms of onychomycosis at baseline. Of the cultures positive for dermatophytes, 11/20 (55\%) showed Trichophyton rubrum, and one patient had Epidermophyton floccosum infection (Fig. 1). All dermatophytomas resolved during the study, with the mean time to resolution of 16 weeks (Fig. 2). All cultures were negative at end of treatment (week 52) and end of study (week 56). 63\% (12/19) subjects and 65\% (13/20) target nails met the primary efficacy endpoint of cure, defined as less than $10 \%$ target nail involvement along with negative fungal culture at end of study (Fig. 3). There were no relapses after cure had been achieved.

\section{Discussion}

No prior study has specifically addressed therapy in patients with dermatophytomas. Our results indicate that efinaconazole $10 \%$ solution has comparable efficacy for toenail infections with dermatophytomas, compared to phase III trials from which dermatophytomas were excluded. Reported complete cure rates (defined as negative $\mathrm{KOH}$ and culture in addition to $0 \%$ nail involve- 


\begin{tabular}{|c|c|c|c|c|c|c|c|}
\hline Subject & $\begin{array}{l}\text { Baseline } \\
\mathrm{KOH}\end{array}$ & $\begin{array}{l}\text { Baseline } \\
\text { culture }\end{array}$ & $\begin{array}{l}\text { Baseline } \\
\text { OASIS }\end{array}$ & $\begin{array}{l}\text { End of } \\
\text { study } \mathrm{KOH}\end{array}$ & $\begin{array}{l}\text { End of study } \\
\text { culture }\end{array}$ & $\begin{array}{l}\text { End of study } \\
\text { OASIS }\end{array}$ & $\begin{array}{l}\text { Weeks to dermato- } \\
\text { phytoma resolution }\end{array}$ \\
\hline 1 & Pos & T. rubrum & 35 & Pos & Neg & 26 & 16 \\
\hline 2 & Neg & Negative ${ }^{\dagger}$ & 26 & Pos & Neg & 19 & 16 \\
\hline $3^{*}$ & Pos & T. rubrum & 14 & Neg & Neg & 0 & 16 \\
\hline 4 & Pos & Negative & 25 & Pos & $\mathrm{Neg}$ & 8 & 16 \\
\hline $5^{*}$ & Pos & T. rubrum & 30 & Neg & $\mathrm{Neg}$ & 0 & 16 \\
\hline 6 & Pos & Negative ${ }^{\dagger}$ & 25 & Neg & Neg & 12 & 16 \\
\hline $7^{*}$ & Pos & Negative & 30 & Neg & Neg & 1 & 16 \\
\hline $8^{*}$ & Pos & T. rubrum & 19 & $\mathrm{Neg}$ & $\mathrm{Neg}$ & 1 & 16 \\
\hline $9^{*}$ & Pos & Negative & 18 & Neg & Neg & 0 & 16 \\
\hline 10 & Pos & T. rubrum & 30 & Pos & Neg & 9 & 16 \\
\hline $11^{*}$ & Pos & T. rubrum & 20 & Neg & Neg & 1 & 24 \\
\hline $12^{*}$ right & Pos & T. rubrum & 26 & $\mathrm{Neg}$ & Neg & 1 & 16 \\
\hline $12^{*}$ left & Pos & T. rubrum & 26 & $\mathrm{Neg}$ & Neg & 1 & 16 \\
\hline $13^{*}$ & Neg & Negative ${ }^{\ddagger}$ & 30 & $\mathrm{Neg}$ & $\mathrm{Neg}$ & 1 & 24 \\
\hline $14^{*}$ & Pos & E. floccosum & 18 & $\mathrm{Neg}$ & $\mathrm{Neg}$ & 0 & 24 \\
\hline $15^{*}$ & Pos & Negative & 16 & Neg & Neg & 0 & 4 \\
\hline 16 & Pos & T. rubrum & 30 & Pos & Neg & 2 & 4 \\
\hline 17 & Pos & T. rubrum & 22 & Neg & $\mathrm{Neg}$ & 2 & 16 \\
\hline $18^{*}$ & Pos & Negative & 18 & $\mathrm{Neg}$ & Neg & 0 & 8 \\
\hline $19 *$ & Pos & T. rubrum & 13 & $\mathrm{Neg}$ & Neg & 0 & 16 \\
\hline
\end{tabular}

Fig. 1. Nineteen subjects with dermatophytomas treated with efinaconazole $10 \%$ solution. ${ }^{*}$ Cure was achieved by the end of study. ${ }^{\dagger}$ Culture positive from another study. ${ }^{\ddagger}$ Enrolled on clinical grounds alone.

ment) were 17.8 and $15.2 \%$ for the multicenter phase III clinical studies using efinaconazole [5]. Additionally, in these studies, 35.7 and $31.0 \%$ achieved treatment success defined as less than or equal to $10 \%$ target nail involvement [5]. Patient motivation with compliance documentation as well as frequent follow-up visits every $4-8$ weeks may also have contributed to higher cure rates than observed in other studies. The low rate of relapse may be attributed to small sample size and short follow-up period.

Similar to past experience, our results indicate that subjects with less severe onychomycosis tended to respond better to therapy. The mean OASIS at baseline for all subjects was 23.55 (range 13-35). Of the subjects who achieved cure, the mean baseline OASIS was 21.38 (range 14-30). Of the subjects who did not achieve cure, mean baseline OASIS score was 27.57 (range 22-35). A significant improvement in OASIS score was seen throughout the study, in parallel with clinical observations. OASIS score of $0-1$ at the end of study correlated with cure. Significant improvement was seen even in some subjects who were not cured at the end of study. For example, subjects 16 and 17 electively terminated therapy at 48 weeks and achieved OASIS scores of 2 but may have seen con-

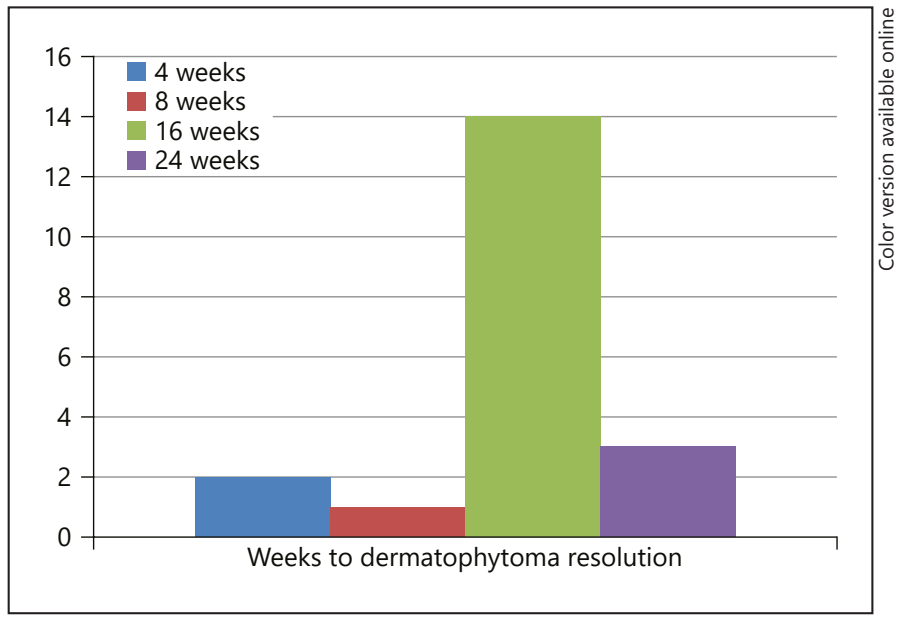

Fig. 2. Dermatophytoma resolution for target nails treated with daily efinaconazole $10 \%$ solution.

tinued improvement if treated for a longer duration (Fig. 1).

Finally, it is important to emphasize that not all successfully treated onychomycosis cases result in a normal appearing nail. Many other causes of nail dystrophy 
Fig. 3. Examples of cures. Left column: baseline, middle column: resolution of dermatophytoma, right column: cure.
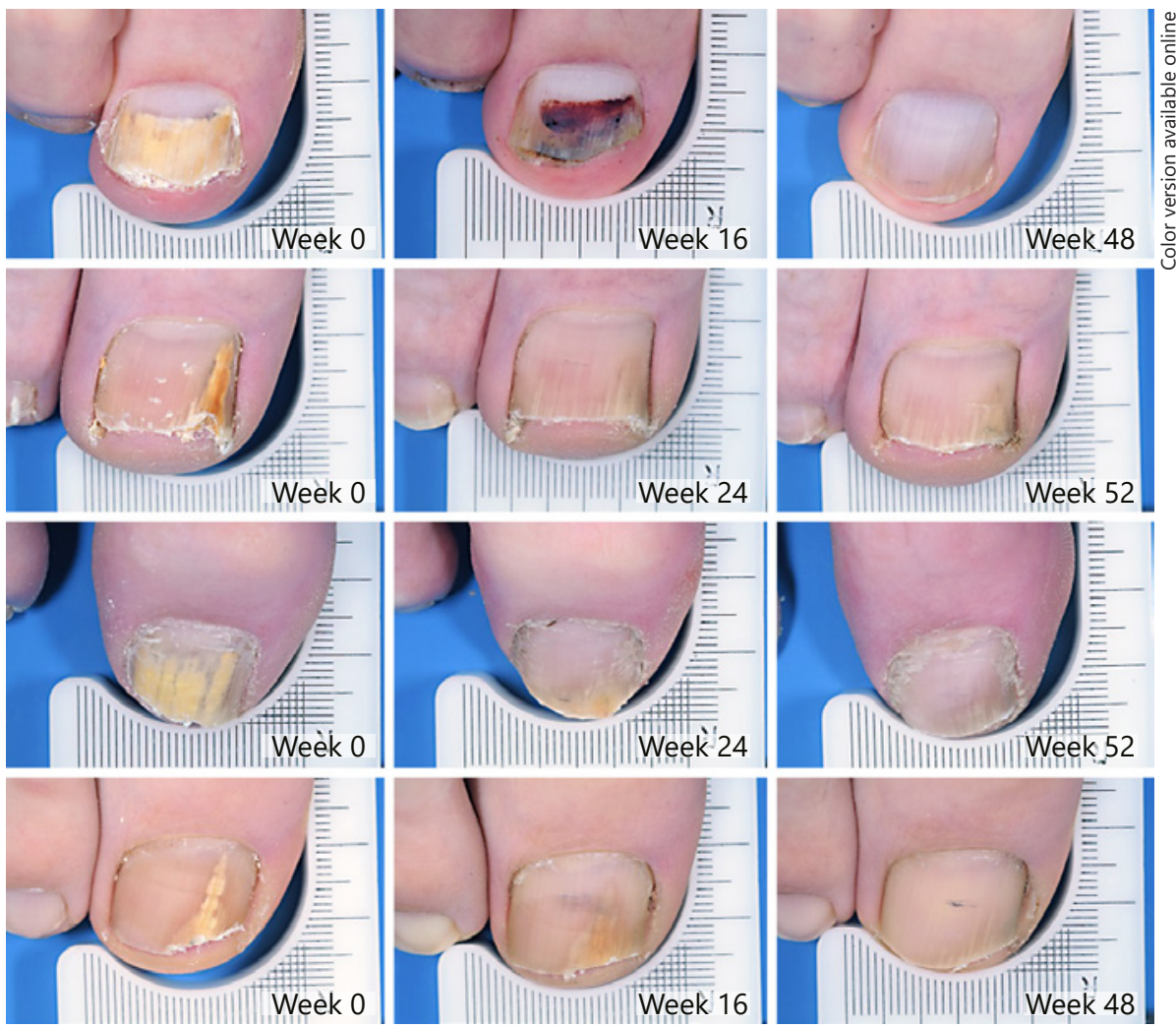

(such as trauma or psoriasis) can predispose a nail to onychomycosis, and the dystrophy may persist despite successful treatment of onychomycosis. Also, in severe cases of onychomycosis, up to $10 \%$ of the nail surface is likely to remain abnormal-appearing after successful treatment.

\section{Conclusions}

The results of this study indicate that efinaconazole $10 \%$ solution is a treatment option for patients with dermatophytoma infections. Patients should be instructed to apply efinaconazole solution on, under, and around the affected nails, and treatment regimen compliance should be emphasized. Given the prevalence of dermatophytomas in patients with onychomycosis, similar investigations using other topical antifungal agents would be useful.

\section{Statement of Ethics}

In the conduction of this study, human subjects have given their informed consent and the study protocol was approved by the institute's committee on human research.

\section{Disclosure Statement}

Boni Elewski, MD: Clinical research support - research funding to University: Abbvie; Boehringer Ingelheim; Celgene; Incyte; Lilly; Merck; Novartis; Pfizer; Regeneron; Sun; Viamet; Valeant. Consultant - received honorarium: Celgene; Lilly; Novartis; Pfizer; Sun; Valeant. The authors Casey Wang, Wendy Cantrell, and Theresa Canavan have no conflicts of interest to declare in this study. 


\section{References}

1 Elewski BE. Onychomycosis: pathogenesis, diagnosis, and management. Clin Microbiol Rev. 1998 Jul;11(3):415-29.

2 Gupta AK, Pillai R. The presence of an air gap between the nail plate and nail bed in onychomycosis patients: treatment implications for topical therapy. J Drugs Dermatol. 2015 Aug; 14(8):859-63.

3 Elewski BE, Rich P, Pollak R, Pariser DM, Watanabe S, Senda $\mathrm{H}$, et al. Efinaconazole $10 \%$ solution in the treatment of toenail onychomycosis: two phase III multicenter, randomized, double-blind studies. J Am Acad Dermatol. 2013 Apr;68(4):600-8.
4 Burkhart CN, Burkhart CG, Gupta AK. Dermatophytoma: recalcitrance to treatment because of existence of fungal biofilm. J Am Acad Dermatol. 2002 Oct;47(4):629-31.

5 Elewski BE, Aly R, Baldwin SL, González Soto RF, Rich P, Weisfeld M, et al. Efficacy and safety of tavaborole topical solution, $5 \%$, a novel boron-based antifungal agent, for the treatment of toenail onychomycosis: results from 2 randomized phase-III studies. J Am Acad Dermatol. 2015 Jul;73(1):62-9.
6 Cantrell W, Canavan T, Elewski B. Report of a case of a dermatophytoma successfully treated with topical efinaconazole $10 \%$ solution. J Drugs Dermatol. 2015 May;14(5):5246.

7 Carney C. A new classification system for grading the severity of onychomycosis: Onychomycosis Area Severity Index Score (OASIS). J Am Acad Dermatol. 2011;64(2): Supplement $1, \mathrm{AB} 92$

8 Scher RK, Tavakkol A, Sigurgeirsson B, Hay RJ, Joseph WS, Tosti A, et al. Onychomycosis: diagnosis and definition of cure. J Am Acad Dermatol. 2007 Jun;56(6):939-44. 